\title{
Atmospheric Loss of Atomic Oxygen during Proton Aurorae on Mars
}

\author{
V. I. Shematovich* \\ Institute of Astronomy, Russian Academy of Sciences, Moscow, 119017 Russia \\ *e-mail: shematov@inasan.rssi.ru
}

Received March 19, 2021; revised April 5, 2021; accepted April 6, 2021

\begin{abstract}
For the first time, the calculations of the penetration of protons of the undisturbed solar wind into the daytime atmosphere of Mars due to charge exchange in the extended hydrogen corona (Shematovich et al., 2021) are used allowing us to determine self-consistently the sources of suprathermal oxygen atoms, as well as their kinetics and transport. An additional source of hot oxygen atoms-collisions accompanied by the momentum and energy transfer from the flux of precipitating high-energy hydrogen atoms to atomic oxygen in the upper atmosphere of Mars-was included in the Boltzmann kinetic equation, which was solved with the Monte-Carlo kinetic model. As a result, the population of the hot oxygen corona of Mars has been estimated; and it has been shown that the proton aurorae are accompanied by the atmospheric loss of atomic oxygen, which is evaluated within a range of (3.5-5.8) $\times 10^{7} \mathrm{~cm}^{-2} \mathrm{~s}^{-1}$. It has been shown that the exosphere becomes populated with a substantial amount of suprathermal oxygen atoms with kinetic energies up to the escape energy, $2 \mathrm{eV}$. The atomic oxygen loss rate caused by a sporadic source in the Martian atmosphere-the precipitation of energetic neutral atoms of hydrogen (H-ENAs) during proton aurorae at Mars-was estimated by the self-consistent calculations according to a set of the Monte-Carlo kinetic models. These values turned out be comparable to the atomic oxygen loss supported by a regular source-the exothermic photochemical reactions (Groeller et al., 2014; Jakosky et al., 2018). It is currently supposed that the atmospheric loss of Mars due to the impact of the solar wind plasma and, in particular, the fluxes of precipitating highenergy protons and hydrogen atoms during solar flares and coronal mass ejections may play an important role in the loss of the neutral atmosphere on astronomic time scales (Jakosky et al., 2018).
\end{abstract}

Keywords: Solar System, Mars, solar wind, hot corona, atmospheric loss, Monte-Carlo kinetic method DOI: $10.1134 / \mathrm{S} 0038094621040079$

\section{INTRODUCTION}

The Sun influences the upper layers of the Martian atmosphere through both the radiation absorbed in the soft X-ray and extreme UV ranges and the solar wind plasma forcing, which results in the formation of the extended neutral corona populated by suprathermal (hot) H, C, N, and $\mathrm{O}$ atoms (see, e.g., Groeller et al., 2014). One of the important results of the Mars Atmosphere and Volatile Evolution (MAVEN) mission was that the Imaging UV Spectrograph (IUVS) observations confirmed the presence of the extended corona composed of hydrogen, carbon, and oxygen atoms (Deighan et al., 2015). The hot corona, in turn, is changed due to the solar wind plasma inflow and the local fluxes of ions picked up from the ionosphere to the planetary exosphere. This inflow leads to the formation of suprathermal atoms (energetic neutral atoms (ENAs)) escaping from the neutral atmosphere of Mars because of the charge exchange with precipitating high-energy ions.
Atomic oxygen escapes from the Martian corona mainly due to the nonthermal processes (see, e.g., Lillis et al., 2015; Shematovich, 2019), the foremost of which are photochemical reactions induced by absorption of solar UV photons in the Martian thermosphere and accompanied by formation of hot (suprathermal) neutral atoms. Suprathermal oxygen atoms populate the so-called hot corona and may escape from the exosphere to open space, if their kinetic energy is high enough (see, e.g., Shematovich, 2019). The dominant photochemical source of suprathermal oxygen atoms is the dissociative recombination of $\mathrm{O}_{2}^{+}$ions with ionospheric electrons. When the recombination occurs in the thermosphere-to-exosphere transition region, upward moving suprathermal atoms will not apparently be scattered backward or slowed down by further collisions to the level below the escape energy and will most likely leave the atmosphere (see, e.g., Fox and Hac, 2009; Lillis et al., 2017). With the instruments onboard the MAVEN spacecraft, the escaping fluxes of neutral oxygen 
atoms cannot be measured directly. Consequently, to estimate the atmospheric losses when interpreting the observations of planetary coronas, numerical models should be used (Shematovich, 2019; Lillis et al., 2017). For example, in the remote sensing of the hot oxygen corona of Mars with the IUVS/MAVEN instrument, the altitude profiles of the hot fraction of oxygen atoms were inferred from the limb scans in the atomic oxygen multiplets at 130.4 and $135.6 \mathrm{~nm}$ (Deighan et al., 2015). To estimate the fraction of escaping oxygen atoms, the values obtained for the content of suprathermal oxygen atoms (Lillis et al., 2017) were compared to the corona models. From this comparison, Jakosky et al. (2018) estimated the loss rate of neutral oxygen as $(3.5-7.0) \times 10^{25}$ atoms per second. Usually, the loss rate of neutral oxygen due to photochemical sources is specified by a representative value of $5 \times 10^{25}$ atoms per second, which is equivalent to the neutral oxygen mass loss with a rate of $1.3 \mathrm{~kg} / \mathrm{s}$.

The planetary atmosphere may escape to open space due to different thermal and nonthermal processes (Shematovich, 2019). In our previous studies (see, e.g., Shematovich and Kalinicheva, 2020; and references therein), we analyzed an additional source of the atomic oxygen escape from the upper atmosphere of Mars induced by precipitation of highenergy protons and hydrogen atoms of the solar wind. This way of losing a neutral upper atmosphere was first proposed in the studies of proton aurorae in the upper terrestrial atmosphere (see, e.g., Shematovich, 2019; and references therein). Though proton auroral events are sporadic, they are indicative of enhanced solar activity and accompanied by rather intense losses of atomic oxygen from the atmosphere of the Earth. Since proton aurorae were recently discovered in observations of the enhanced Ly- $\alpha$ emission of atomic hydrogen (Ritter et al., 2018; Deighan et al., 2018), some researchers attempted to estimate the contribution of atmospheric sputtering during proton auroral events at Mars to the atomic oxygen loss rate (see, e.g., Shematovich and Kalinicheva, 2020; and references therein). These attempts have a particular importance, because this process was ignored in the recent analysis of the atmospheric loss of Mars based on the MAVEN data (Jakosky et al., 2018).

In this paper, we study the processes of kinetics and transport of hot oxygen atoms in the upper atmosphere of Mars, which are during aurorae on Mars caused by precipitation of hydrogen atoms from the solar wind. Since the proton auroral events are observed on the dayside of Mars (Hughes et al., 2019) and induced by the fluxes of high-energy hydrogen atoms penetrating into the atmosphere (Deighan et al., 2018), to calculate the source function of suprathermal oxygen atoms, we use the Monte-Carlo kinetic models (Shematovich et al., 2019) developed before. These models make it possible to analyze the charge-exchange process for solar-wind protons in the extended hydrogen corona of Mars and obtain the spectra of hydrogen atoms penetrating into the atmosphere through the boundary of the induced magnetosphere of Mars (Shematovich et al., 2021). Further, these spectra of hydrogen atoms serve as an upper boundary condition for the Monte-Carlo kinetic model of precipitation of energetic hydrogen atoms into the upper atmosphere (Shematovich et al., 2019), which allows the characteristics of proton auroral events on Mars to be modeled. Specifically, we obtain the formation rate and energy spectra of suprathermal oxygen atoms produced in elastic and inelastic collisions between atmospheric oxygen atoms and hydrogen atoms penetrated into the atmosphere. And, finally, these formation rates of suprathermal oxygen atoms are used as a source for the Monte-Carlo kinetic model (Shematovich and Kalinicheva, 2020) to study the kinetics and transport of suprathermal oxygen atoms in the upper atmosphere of the planet. As a result, we derive the kinetic-energy distribution functions for suprathermal oxygen atoms in the thermosphere-to-exosphere transition region. It has been found that the proton auroral events observed at the dayside of Mars are accompanied by forming a substantial amount of suprathermal oxygen atoms with kinetic energies up to the escape energy, $2 \mathrm{eV}$. In other words, in addition to the photochemical sources, a hot fraction of the oxygen corona of Mars is formed and an additional nonthermal flux of atomic oxygen escaping from the Martian atmosphere is generated. In our previous studies (see, e.g., Shematovich and Kalinicheva, 2020; and references therein), we used only simplified estimates of the flux of hydrogen atoms penetrating into the atmosphere and their approximated energy spectra as an upper boundary condition. This paper is the first one based on the calculation results for the penetration of undisturbed solar-wind protons into the illuminated atmosphere of Mars due to the charge exchange in the extended hydrogen corona (Shematovich et al., 2021). This allows us to determine selfconsistently the sources of suprathermal oxygen atoms, as well as their kinetics and transport, and to estimate the population of the hot oxygen corona of Mars and the atmospheric losses of atomic oxygen during proton auroral events. Though proton aurorae are sporadic events, the recent MAVEN observations showed that the precipitation-induced flux of escaping hot oxygen atoms may become dominant under conditions of extreme solar events-solar flares and coronal mass ejections (Deighan et al., 2015; Lee et al., 2018; Jakosky et al., 2015, 2018). The analysis of the atomic oxygen loss due to atmospheric sputtering during proton aurorae on Mars should be taken into account especially when studying the climate evolution of the planet on geological time scales. 


\section{FORMATION OF HOT OXYGEN ATOMS DURING PROTON AURORAE}

One of the interesting results of the MAVEN space mission was that a small fraction of protons with energies close to the solar wind energy was observed with the Solar Wind Ion Analyzer (SWIA) at altitudes of roughly $150-250 \mathrm{~km}$ in the atmosphere of Mars (Halekas et al., 2015). According to these measurements, the velocity of this fraction almost coincides with that of the solar wind observed. From the previous studies, it was known that some fraction of the solar wind may interact with the extended corona of Mars. After the charge exchange with hydrogen atoms in this corona, a certain portion of protons of the impacting solar wind may gain an electron and become energetic neutral atoms of hydrogen (H-ENAs). Since these particles are neutral, they easily penetrate through the induced magnetosphere boundary (IMB) of Mars and get into denser regions of the neutral atmosphere.

The extended hydrogen corona was observed more than once in the Ly- $\alpha$ line of the scattered solar UV radiation by the instruments onboard the Mars Express spacecraft (Chaufray et al., 2008), the Hubble Space Telescope (Clarke et al., 2014), and the MAVEN spacecraft (Chaffin et al., 2018). These observations revealed substantial seasonal changes of the atomic hydrogen concentration along the view axis in the Martian corona. The SWIA/MAVEN measurements, which were carried out for a full Martian year, allow us to say confidently that, in the Martian corona, the atomic hydrogen column density may vary by an order of magnitude (Halekas, 2017). The analysis of the observed hydrogen density distributions suggests that the escape of hydrogen cannot take place only at the expense of molecular hydrogen. This notion is confirmed by the detection of water vapor at altitudes of 40$100 \mathrm{~km}$ by the SPICAM/MEX instrument during observations of the solar eclipse (Maltagliati et al., 2013; Fedorova et al., 2018). These studies show that water is not so effectively kept in the cold trap of the mesosphere, as previously supposed. New data on the vertical distribution of water vapor in the upper atmosphere of Mars were obtained in observations of the Atmospheric Chemistry Suite (ACS) onboard the ExoMars Trace Gas Orbiter (Vandaele et al., 2019; Fedorova et al., 2020). They detected considerable rapid seasonal intrusions of water and ice particles into the upper atmospheric layers, which resulted in an increase in the water content in the corona. Depending on the abundance and altitude location of water vapor and ice particles transported to the altitudes up to $100 \mathrm{~km}$ (Vandaele et al., 2019; Fedorova et al., 2020), this effect may induce seasonally variable changes in the hydrogen column density in the Martian corona even by an order of magnitude (Chaffin et al., 2017; Heavens et al., 2018).
With the Monte-Carlo kinetic model (thereafter, MCKM-1), Shematovich et al. (2021) numerically analyzed the influence of changes observed in the density of the extended hydrogen corona of Mars on the charge-exchange efficiency of undisturbed solarwind protons. They determined the parameters and characteristics of the precipitation of $\mathrm{H}$-ENAs, which are formed in the charge exchange, into the illuminated atmosphere of Mars. This made it possible to study in detail the proton auroral events on Mars. It was found that, for the observed variations in the atomic hydrogen column density in the Martian corona, the charge-exchange efficiency changes within $4-8 \%$, while the energy spectrum of hydrogen atoms penetrating through the IMB into the Martian atmosphere is identical to that of undisturbed protons of the solar wind.

Energetic atoms of hydrogen (H-ENAs), penetrating into the illuminated atmosphere, collide with the main atmospheric components, which are accompanied by the momentum and kinetic energy transfer in elastic and inelastic collisions and the processes of ionization and electron detachment (i.e., ionization of an impacting hydrogen atom). Consequently, the interaction of the precipitating $\mathrm{H}$-ENA fluxes with the main neutral components of the atmospheric gas should be considered as a cascade process, which is accompanied by the formation of an increasing amount of translationally and internally excited particles of the ambient atmospheric gas. To analyze the penetration of the flux of high-energy $\mathrm{H}^{+} / \mathrm{H}$ particles of the solar wind into the upper atmosphere of Mars, we use a modified version of the Monte-Carlo kinetic model (thereafter, MCKM-2) developed earlier for the data analysis of the measurements carried out with the Analyzer of Space Plasma and Energetic Atoms (ASPERA-3) and SWIA instruments onboard the Mars Express (MEX) and MAVEN spacecraft, respectively (Shematovich et al., 2019, 2021).

For the analyzed case of proton aurorae at Mars, the H-ENA precipitation process may be considered as an additional source of suprathermal oxygen atoms (Shematovich and Kalinicheva, 2020), which are formed due to the atmospheric sputtering of the main component of the upper atmosphere, atomic oxygen, by H-ENAs. Consequently, one of the results of penetration of the precipitating H-ENA flux from the solar-wind into the upper atmospheric layers is the formation of suprathermal oxygen atoms $\mathrm{O}_{\mathrm{h}}$ by transferring the momentum in elastic and inelastic collisions with atmospheric oxygen $\mathrm{O}_{\mathrm{th}}$ :

$$
\mathrm{H}^{+}(\mathrm{H})+\mathrm{O}_{\mathrm{th}} \rightarrow \mathrm{H}^{+}(\mathrm{H})+\mathrm{O}_{\mathrm{h}} .
$$

Fresh suprathermal oxygen atoms lose the excess energy in collisions with other particles in the atmosphere and propagate into the thermosphere-to-exo- 
sphere transition region (Shematovich and Kalinicheva, 2020). Their kinetics and transport are described by the Boltzmann kinetic equation for the velocity distribution functions $f_{\mathrm{Oh}}(\mathbf{r}, \mathbf{v})$ and $f_{\mathrm{M}}(\mathbf{r}, \mathbf{v})$ for hot oxygen atoms and components of the ambient atmospheric gas, respectively (see, e.g., Shematovich and Kalinicheva, 2020; and references therein). It is supposed that the atmospheric gas is characterized by the velocity distribution described by the locally equilibrium Maxwell function.

The Monte-Carlo kinetic method is an effective tool to study complex kinetic systems in the stochastic approximation (Shematovich, 2019). In the numerical realization of the abovementioned model of the kinetics and transport of the flux of hydrogen atoms precipitating from the solar wind plasma (MCKM-2), the statistics of collisions (Eq. (1)), which are accompanied by the formation of suprathermal oxygen atoms, is accumulated. Based on this statistics, the source function $Q_{\mathrm{Oh}}(E)$ is determined: $\mathrm{H}(E)+\mathrm{O}_{\text {th }} \rightarrow \mathrm{H}^{+}$ $\left(E^{\prime}<E\right)+\mathrm{O}_{\text {sth }}\left(E^{\prime \prime}=E-E\right)$, where $E$ and $E^{\prime}$ are the kinetic energies of $\mathrm{H}$ particles before and after a collision. The function $Q_{\mathrm{Oh}}(E)$ specifies the formation rate of suprathermal oxygen atoms in precipitation of highenergy $\mathrm{H}$ particles, and it is used as input data for the Monte-Carlo kinetic model of collisions and transport of hot oxygen atoms in the transition region (MCKM$3)$. The output data of the MCKM-2 are the volume rates of collision-induced formation of suprathermal atoms (Eq. (1)) and the distribution function of their kinetic energy in the considered transition region of the Martian atmosphere. This numerical model corresponds to a microscopic level of the gas state description in the planetary corona (Shematovich, 2019); and the local mean time and the local mean free path of hot particles at the lower boundary of the transition region, where the ambient atmospheric gas is denser, are assumed as characteristic time and space scales. Since we are mostly focused on calculating the population of suprathermal oxygen atoms in the transition region of the upper atmosphere of Mars, the lower limit for the energy of suprathermal atoms is specified in the model by a value of $0.08 \mathrm{eV}$, which corresponds to a temperature of around $180-200 \mathrm{~K}$ in the exosphere for a low level of solar activity.

The considered region of the Martian atmosphere is bounded by altitudes of 80 and $500 \mathrm{~km}$. As a lower boundary, we consider the relatively dense thermosphere, where hot particles quickly lose the excess kinetic energy in collisions with thermal molecules of carbon dioxide and oxygen. The upper boundary is assumed to be above the exobase $(\sim 190 \mathrm{~km})$, where the gas flow becomes a free-molecular one. The considered region is divided into cells with a step of $5 \mathrm{~km}$. In each of the cells, the atmospheric gas, which is composed of $\mathrm{CO}_{2}$ and $\mathrm{N}_{2}$ molecules and $\mathrm{O}$ atoms, is represented by model particles with the concentration and temperature specified in accordance with the calculation results by Fox and Hac (2009). According to the physical model, in each of the cells, model particles, acting as sources of suprathermal oxygen atoms in process (1), are born; they move in the gravitational field of Mars and produce secondary suprathermal particles in collisions with the atmospheric gas. Since the simulations are performed at the molecular level, the detailed statistics are accumulated for the spatial distribution of suprathermal oxygen atoms by velocities (kinetic energies) and the energy spectra for the fluxes of particles populating the hot oxygen corona, as well as those escaping from the Martian atmosphere.

\section{MODEL RESULTS}

We calculate the source function for suprathermal oxygen atoms with the earlier developed Monte-Carlo kinetic model (Shematovich et al., 2019). The latter was used to study the charge-exchange process for solar-wind protons in the extended hydrogen corona of Mars and to obtain the spectra of hydrogen atoms penetrating into the atmosphere through the IMB of Mars (Shematovich et al., 2021). In the following, the obtained spectra of hydrogen atoms are used as an upper boundary condition for the MCKM-2 of the precipitation of energetic hydrogen atoms into the upper atmosphere, which allows the characteristics of proton aurorae on Mars to be numerically estimated (Shematovich et al., 2019). In particular, these simulations yielded the formation rates and the energy spectra of oxygen atoms with suprathermal energies caused by elastic and inelastic collisions (1) between atmospheric oxygen atoms and hydrogen atoms penetrating into the atmosphere. Finally, the formation rates of suprathermal oxygen atoms obtained in this way were used as a source function for the MCKM-3 (Shematovich and Kalinicheva, 2020) to study the kinetics and transport of suprathermal oxygen atoms in the upper atmosphere of the planet. The present study is the first one, which is based on the calculation results for the penetration of undisturbed solar-wind protons into the illuminated atmosphere of Mars due to the charge exchange in the extended hydrogen corona (Shematovich et al., 2021). This allows us to calculate self-consistently both the sources of suprathermal oxygen atoms and their kinetics and transport.

To estimate the contribution of an additional source of suprathermal oxygen atoms during proton auroral events-collisions (1) with transferring the excess energy from precipitating high-energy hydrogen atoms to thermal oxygen atoms-to the formation of the extended hot corona of Mars, we use, analogously to the paper by Shematovich et al. (2021), models A and B with the following calculation logistics. 

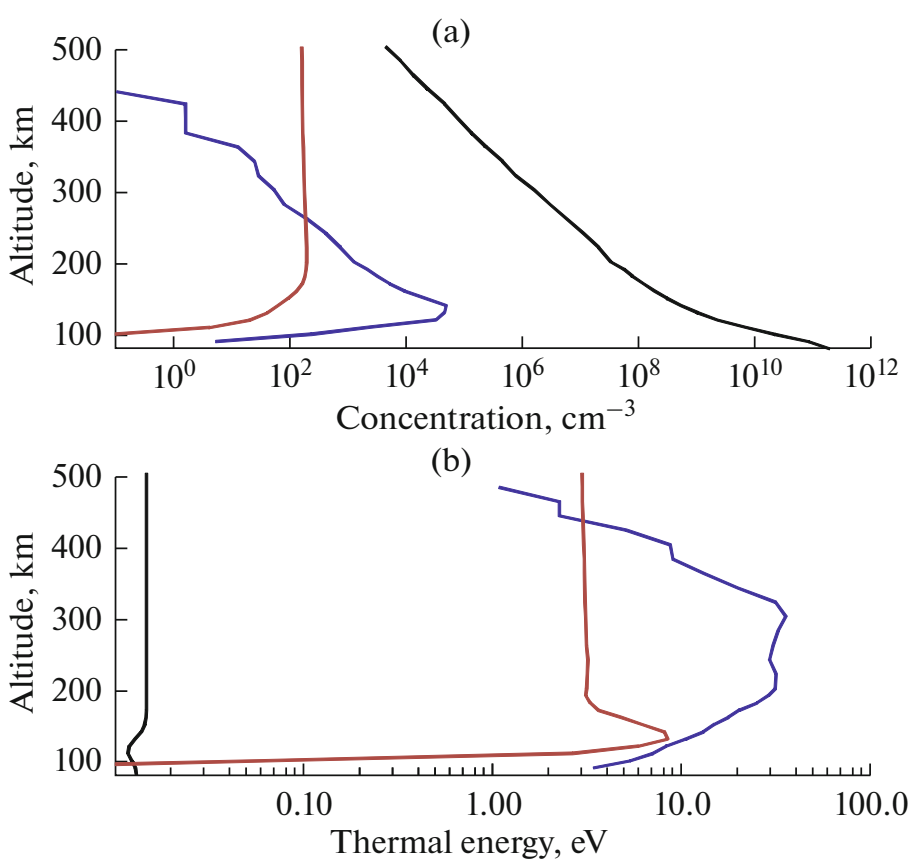

Fig. 1. (a) Altitude profiles for the concentration of atmospheric oxygen (black line) and suprathermal oxygen (brown line) calculated with the MCKM-3. The blue line shows the formation rate of suprathermal oxygen atoms due to precipitation of energetic hydrogen atoms. (b) Altitude profiles for the mean kinetic energy of atmospheric oxygen (black line) and suprathermal oxygen (brown line) obtained in calculations. The blue line shows the mean kinetic energy of suprathermal oxygen atoms formed due to the precipitation of hydrogen atoms induced by the charge exchange of solar-wind protons in the extended hydrogen corona of Mars. The calculations were made for the basic model with the IMB at an altitude of $820 \mathrm{~km}$.

- Model A is a basic one, within which the temperature and density profiles of the main components of the upper atmosphere, $\mathrm{CO}_{2}, \mathrm{CO}$, and $\mathrm{O}$, were taken from the model by Fox and Hac (2009), that corresponds to a low level of solar activity. The distribution of hydrogen atoms in the extended hot corona of Mars was specified by the Chamberlain model of the planetary atmosphere. Its parameters were chosen as follows: the exobase altitude was $h_{\mathrm{exo}}=200 \mathrm{~km}$ and the temperature and the density of atomic hydrogen were $T\left(h_{\text {exo }}\right)=179 \mathrm{~K}$ and $n_{\mathrm{H}}\left(h_{\text {exo }}\right)=1.48 \times 10^{6} \mathrm{~cm}^{-3}$ in accordance with the results by Chaffin et al. (2018). Further, as a boundary condition at an altitude of $3000 \mathrm{~km}$ for the MCKM-1 (Shematovich et al., 2021), we used the energy flux and the energy spectrum of protons from the undisturbed solar wind, which was measured by the SWIA/MAVEN instrument for MAVEN orbit on February 27, 2015 (Halekas et al., 2015). The spectra of hydrogen atoms calculated with the MCKM-1 were assumed as an upper boundary condition for the MCKM-2 (Shematovich et al., 2019) that simulates the precipitation of energetic hydrogen atoms into the upper atmosphere through the IMB at an altitude of $820 \mathrm{~km}$. This made it possible to calculate the altitude profiles of the volume formation rate and the energy spectra of oxygen atoms produced in collisions (1) with suprathermal energies. These characteristics served as source functions in the MCKM-3 (Shema- tovich, 2019), with which the kinetics and transport of suprathermal oxygen atoms in the upper atmosphere of the planet were studied.

-With model B, the variation in the hydrogen column density in the Martian corona was taken into account. Namely, under the same parameters of the exobase, the concentration of hydrogen atoms $n_{\mathrm{H}}\left(h_{\mathrm{exo}}\right)$ increased twofold, which corresponds to the position of the IMB at an altitude of $1260 \mathrm{~km}$ (for details, see (Shematovich et al., 2021)). The other calculations of the distribution of suprathermal oxygen atoms in the upper atmosphere of Mars were performed in the same way as for Model A.

The calculations were made for the zero zenith angle of the Sun. The results of calculations according to the above-described models $\mathrm{A}$ and $\mathrm{B}$ are shown in Figs. 1-4. In numerical realizations of the MonteCarlo kinetic models, the statistics of distributions of superthermal and suprathermal atoms of hydrogen and oxygen by kinetic energy was accumulated in all space cells. Namely, in the MCKM-1, it is the flux and the energy spectrum of hydrogen atoms penetrating through the IMB into the upper atmosphere of Mars; in the MCKM-2, it is the volume formation rates of suprathermal oxygen atoms and their distribution by kinetic energy; and in the MCKM-3, it is the stationary distribution of suprathermal oxygen atoms and the 

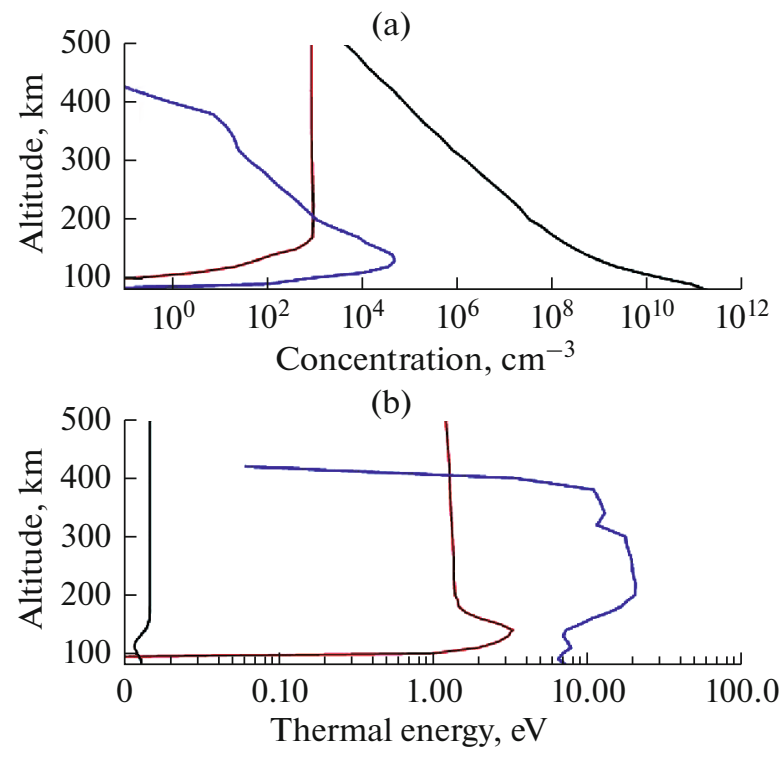

Fig. 2. As in Fig. 1, but the calculations were made for the model with the double hydrogen content in the extended corona of Mars and, consequently, with the IMB at an altitude of $1260 \mathrm{~km}$.

energy spectrum of the escaping flux in the thermosphere-to-exosphere transition region of the Martian atmosphere.

The altitude profiles of the formation rate and the mean kinetic energy of suprathermal oxygen atoms produced in collisions (1) are shown by blue lines in Figs. 1 and 2 for models A and B, respectively. In the same diagrams, the brown lines present the concentration of suprathermal oxygen atoms and their mean kinetic energy calculated with the MCKM-3, while the black lines present the altitude profiles for the concentration and the thermal energy of atmospheric oxygen atoms according to the data by Fox and Hac (2009). Panels (a) and (b) of Figs. 1 and 2 show the concentration of different fractions of atomic oxygen and the mean kinetic energy, respectively.

As is seen from Figs. 1 and 2, the formation rate of suprathermal oxygen atoms is higher in model B, which leads to a higher concentration of these atoms in the Martian corona. However, the mean kinetic energy of suprathermal atoms in model B is somewhat lower than that in model A. This distribution by altitude and energy of the source of suprathermal oxygen atoms induced by the H-ENA precipitation is determined by the charge-exchange efficiency for solarwind protons, which is higher in the denser hydrogen corona of Mars, corresponding to the model with a double hydrogen content, than that in model A (for details, see Shematovich et al. (2021)). We note that, as is seen from the comparison of the altitude profiles of hot oxygen atoms (brown lines in panels (a) of Figs. 1 and 2) and the thermal ones (black lines in the same diagrams), the characteristic scale height for the distribution of suprathermal oxygen atoms is significantly higher than the same quantity for thermal atoms. This is caused by the substantially higher mean kinetic energy of suprathermal oxygen atoms.

In panels (a) of Figs. 3 and 4, we present the results of the MCKM-2 calculations according to models A and $\mathrm{B}$ for the kinetic-energy distribution (normalized on unity) of oxygen atoms formed due to the precipitation of hydrogen atoms induced by the charge exchange of solar-wind protons in the extended hydrogen corona of Mars. In panels (b) of Figs. 3 and 4, we present the results of the MCKM-3 calculations of the stationary energy distribution function (EDF) of suprathermal oxygen atoms for proton aurorae corresponding to models A and B. The EDFs were calculated for an altitude of $140 \mathrm{~km}$, which corresponds to the highest formation rate of fresh suprathermal oxygen atoms induced by precipitation of high-energy hydrogen atoms.

In Figs. 3 and 4 the vertical line indicates the energy of atomic oxygen escaping from the Martian atmosphere, $\sim 2 \mathrm{eV}$. The distribution of oxygen atoms formed in collisions (1) due to precipitation of $\mathrm{H}$-ENAs shows that this source causes the emergence of atoms with the kinetic energy up to $100 \mathrm{eV}$ in the atmosphere, while their distribution is extremely nonequilibrium. From the calculation results for the stationary EDFs shown in panels (b) of Figs. 3 and 4, it is seen that the suprathermal energy range below $2 \mathrm{eV}$ is substantially populated and formed due to elastic collisions with thermal oxygen atoms, which are those introducing the additional component (brown lines in Figs. 1 and 2) to the photochemical fraction of the hot corona of Mars (Groeller et al., 2014). The calculations revealed the essentially nonequilibrium behavior of the EDFs of oxygen atoms as compared to those of the thermal fraction of the oxygen corona. This is caused by the presence of a substantial fraction of oxygen atoms in the suprathermal energy range $(>1.0 \mathrm{eV})$ and the predominance of suprathermal oxygen atoms at altitudes above the exobase (i.e., at $180-200 \mathrm{~km}$ ) in the hot corona, which were produced by reaction (1) and transported there from the lower layers of the thermosphere. The result is that the number of particles with energies exceeding the escape energy becomes larger than the number of such particles at altitudes below the exobase, where suprathermal particles effectively lose the excess kinetic energy in frequent collisions with particles of the neutral atmospheric gas-oxygen atoms and carbon dioxide molecules. Moreover, the precipitation processes induce the formation of extended tails in the distribution of oxygen atoms in the suprathermal energy range $(E>10 \mathrm{eV})$, i.e., they serve as a source of energetic oxygen atoms (O-ENAs) in the space around Mars. These particles 

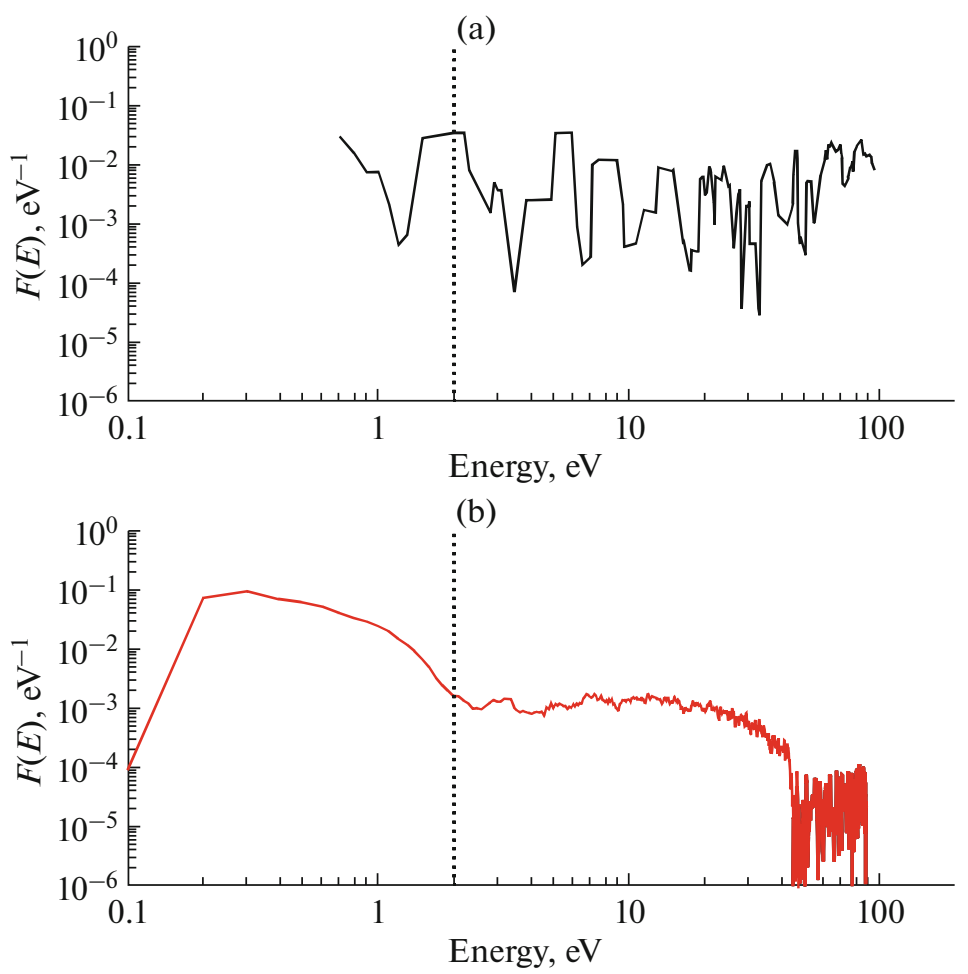

Fig. 3. The kinetic-energy distribution functions normalized to unity (a) for oxygen atoms formed in collisions (1) induced by the precipitation of hydrogen atoms due to the charge exchange of solar-wind protons in the extended hydrogen corona of Mars and (b) for the calculated steady-state fraction of suprathermal oxygen atoms. The calculations were made according to the basic model A with the IMB at an altitude of $820 \mathrm{~km}$; the results are shown for an altitude of $140 \mathrm{~km}$, where the highest values of the formation rate of suprathermal oxygen atoms due to precipitation of H-ENAs is observed. The vertical line marks the escape energy for the Martian atmosphere.

were partially detected by the ASPERA-3 instrument onboard the Mars Express spacecraft.

In Figs. 5 and 6, we present the results of the MCKM-3 calculations of the upward (solid lines) and downward (dashed lines) fluxes of suprathermal oxygen atoms at altitudes of 140 and $220 \mathrm{~km}$ (in panels (a) and (b), respectively) in the upper atmosphere of Mars for models A and B, respectively. At the $140-\mathrm{km}$ altitude, the rate of forming suprathermal oxygen atoms due to precipitation of H-ENAs is highest, while the $220-\mathrm{km}$ level is in the Martian exosphere, from where oxygen atoms with suprathermal energies escape. Figure 7 shows the altitude profiles of the energy-integrated upward flux of suprathermal oxygen atoms for models A (black line) and B (brown line).

In fact, the upward fluxes of suprathermal oxygen atoms, which are shown in panels (b) of Figs. 5 and 6 , make it possible to estimate the fluxes of atomic oxygen escaping from the Martian atmosphere during proton aurorae. For this, the upward flux of oxygen atoms should be integrated over energy starting from the escape energy marked by a vertical line in the diagrams. As a result, the following estimates were obtained for the escaping fluxes: $5.8 \times 10^{7}$ and $3.5 \times$ $10^{7} \mathrm{~cm}^{-2} \mathrm{~s}^{-1}$ for models $\mathrm{A}$ and $\mathrm{B}$, respectively. Thus, collisions between thermal oxygen atoms and precipi-
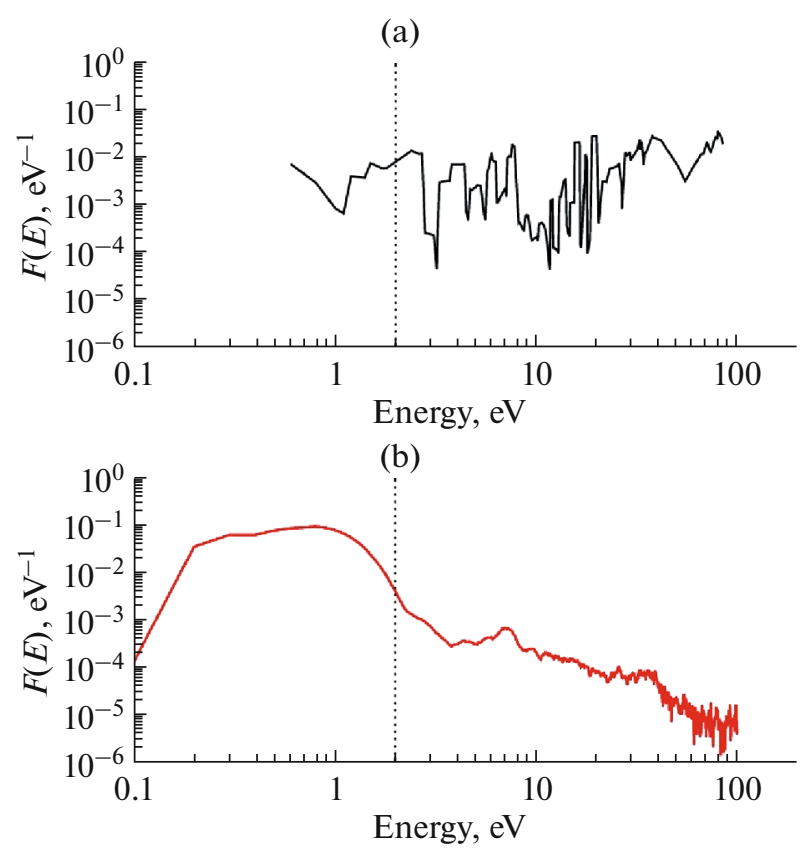

Fig. 4. As in Fig. 3, but the curves present the results of calculations according to model B for an altitude of $140 \mathrm{~km}$, where the highest values of the formation rate of suprathermal oxygen atoms due to precipitation of $\mathrm{H}$-ENAs is observed. The vertical line marks the escape energy for the Martian atmosphere. 

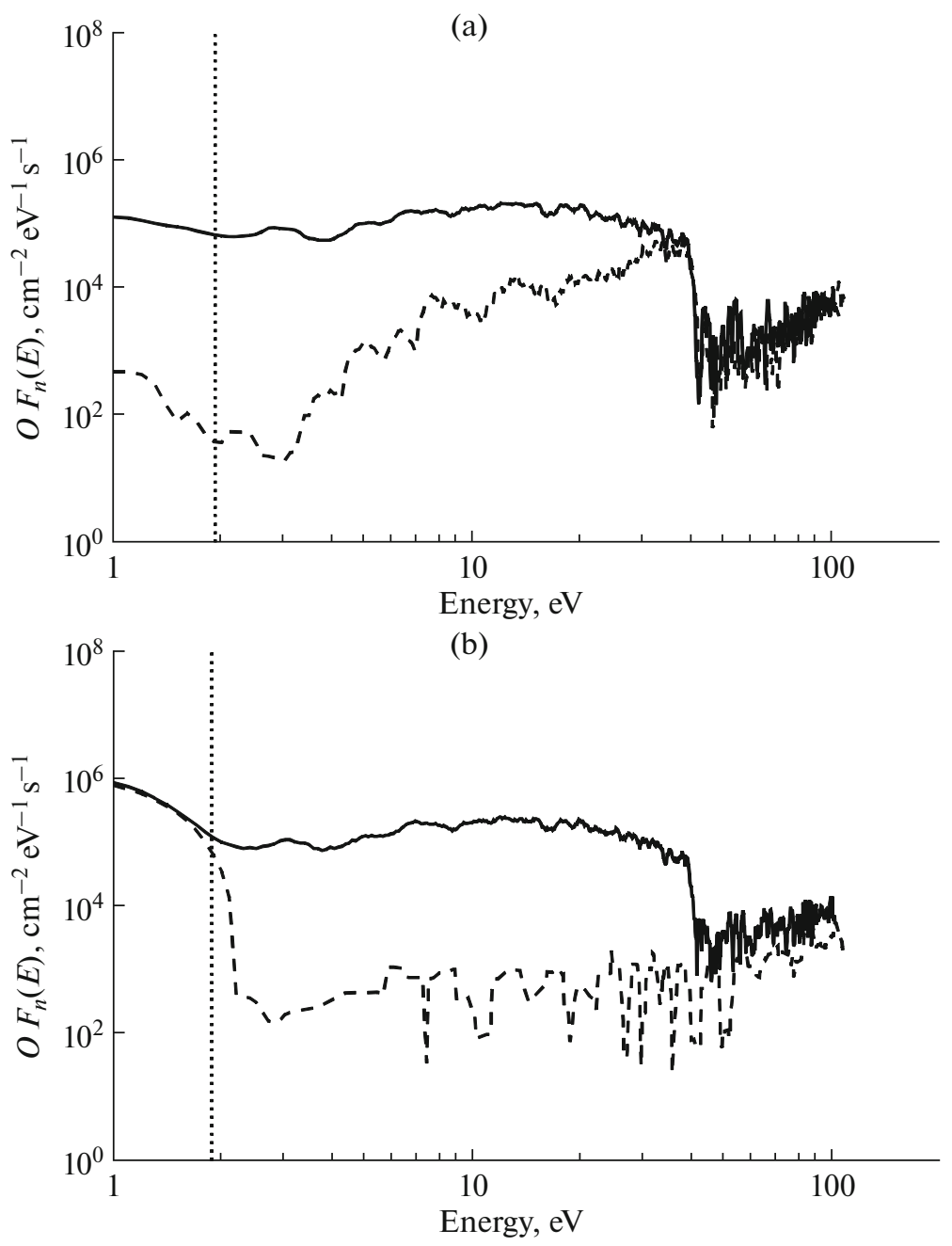

Fig. 5. The upward (solid lines) and downward (dashed lines) fluxes of suprathermal oxygen atoms in the upper atmosphere of Mars at altitudes of $140 \mathrm{~km}$ (a) and $220 \mathrm{~km}$ (b) calculated with the MCKM-3 for the basic model A. At the 140-km altitude, the rate of forming suprathermal oxygen atoms due to precipitation of H-ENAs is highest, while the 220-km level is in the Martian exosphere, from where oxygen atoms with suprathermal energies escape. The vertical line marks the escape energy for the Martian atmosphere.

tating high-energy hydrogen atoms, i.e., $\mathrm{Q}_{\mathrm{Oh}}$ : $\mathrm{H}(E)+$ $\mathrm{O}_{\mathrm{th}} \rightarrow \mathrm{H}\left(E^{\prime}<E\right)+\mathrm{O}_{\text {sth }}\left(E^{\prime \prime}=E-E^{\prime}\right)$, serves as an additional source in proton aurorae and leads to the loss of atomic oxygen from the Martian atmosphere, the escaping flux of which ranges within $(3.5-5.8) \times$ $10^{7} \mathrm{~cm}^{-2} \mathrm{~s}^{-1}$.

From our earlier studies (see, e.g., the paper by Groeller et al. (2014) and references therein), it is known that the nonthermal flux of oxygen, escaping due to exothermic photochemistry, varies with the solar activity level in an interval of $(0.5-5.0) \times 10^{7} \mathrm{~cm}^{-2} \mathrm{~s}^{-1}$. At the same time, in the previous studies of the contribution of precipitation into the atmospheric losses, the following estimates were obtained: (i) the flux of oxygen atoms, escaping due to precipitation of protons and hydrogen atoms from the solar wind, varies in an interval of $(0.7-28.0) \times 10^{5} \mathrm{~cm}^{-2} \mathrm{~s}^{-1}$ for the spectra of precipitating protons measured by the ASPERA-3/MEX instrument under conditions of a low level of solar activity and the spectra of precipitating hydrogen atoms derived by the hybrid modeling of the interaction between the solar wind plasma and the Martian corona; (ii) the flux of oxygen atoms escaping due to precipitation of protons and hydrogen atoms from the solar wind, which are presented by the energy spectra measured locally with the MAVEN/SWIA instrument (Halekas et al., 2015), varies in an interval of (0.315.3) $\times 10^{7} \mathrm{~cm}^{-2} \mathrm{~s}^{-1}$ (e.g., Shematovich and Kalinicheva, 2020; and references therein). The estimates obtained here for the atmospheric loss of atomic oxygen during aurorae on Mars are close to those derived by Shematovich and Kalinicheva (2020); however, the present calculations are the first ones that were carried out self-consistently. Namely, we used 

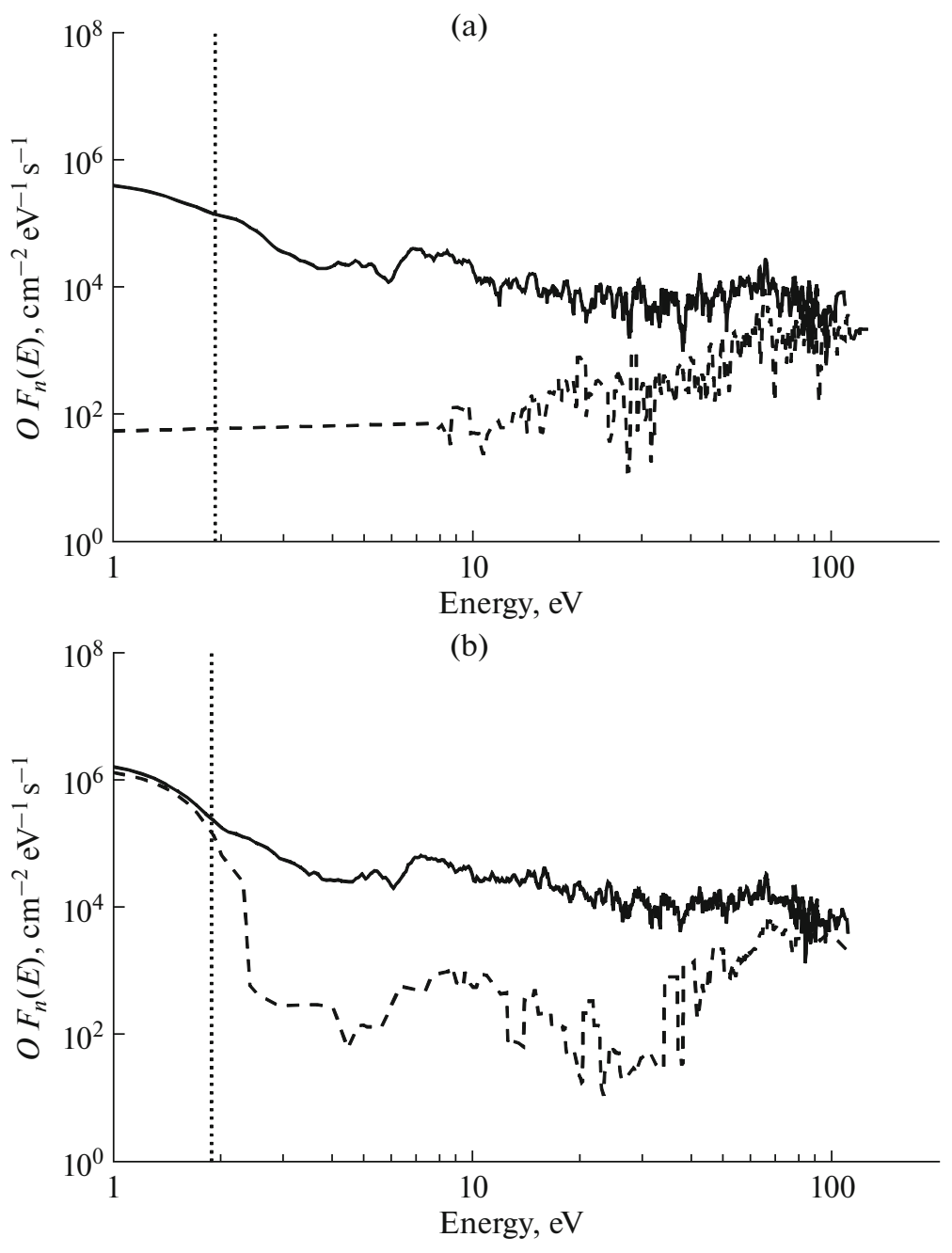

Fig. 6. As in Fig. 5, but the results of calculations according to model B for the upward (solid lines) and downward (dashed lines) fluxes of suprathermal oxygen atoms are presented.

only the energy spectra of solar-wind protons before their interaction with the hydrogen corona, which were measured by the MAVEN/SWIA instrument (Halekas et al., 2015), while the main processes that take place in proton aurorae-the charge exchange of solar-wind protons in the extended hydrogen corona of Mars, which is accompanied by precipitation of $\mathrm{H}-$ ENAs into the neutral atmosphere and formation of a flux of suprathermal oxygen atoms escaping from the atmosphere-were calculated with a set of MonteCarlo kinetic models (Shematovich et al., 2019, 2021). Consequently, this suggests that the precipitationinduced flux of escaping oxygen atoms may be dominant under conditions of extreme solar events-solar flares and coronal mass ejections-when the energy fluxes of precipitating protons and hydrogen atoms may be several orders of magnitude higher than the values typical of the low solar activity conditions (Halekas et al., 2015; Jakosky et al., 2018).

\section{CONCLUSIONS}

In this paper, we considered the processes of formation, kinetics, and transport of suprathermal oxygen atoms in the thermosphere-to-exosphere transition region during proton aurorae in the illuminated atmosphere of Mars, which are induced by precipitation of energetic hydrogen atoms. An additional source of hot oxygen atoms-collisions (1) accompanied by the momentum and energy transfer from the flux of precipitating high-energy particles $\mathrm{H} / \mathrm{H}^{+}$to atomic oxygen in the upper atmosphere of Mars, i.e., $Q_{\mathrm{Oh}}: \mathrm{H}(E)+\mathrm{O}_{\mathrm{th}} \rightarrow \mathrm{H}\left(E^{\prime}<E\right)+\mathrm{O}_{\mathrm{h}}\left(E^{\prime \prime}=E-E\right)$-was included in the Boltzmann kinetic equation, which was solved with the Monte-Carlo kinetic model (e.g., Shematovich and Kalinicheva, 2020; and references therein). As a result, the population of the hot oxygen corona of Mars was estimated; and we showed that the proton aurorae are accompanied by the atmospheric losses of atomic oxygen ranging within $(3.5-5.8) \times 10^{7} \mathrm{~cm}^{-2} \mathrm{~s}^{-1}$. 


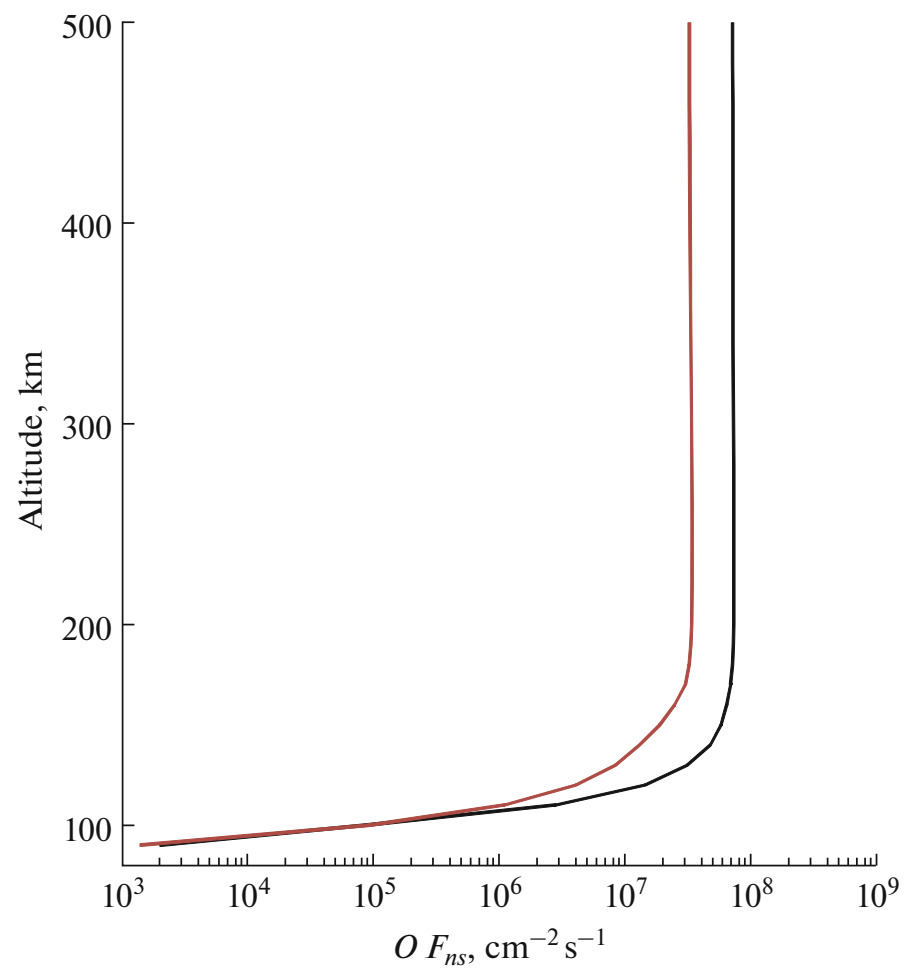

Fig. 7. The altitude profiles for the upward flux of suprathermal oxygen atoms calculated for models A (black line) and B (brown line).

It was shown that the exosphere is populated by a substantial amount of suprathermal oxygen atoms with kinetic energies up to the escape energy, $2 \mathrm{eV}$, i.e., the hot oxygen corona of Mars is in addition to the photochemical sources. With the self-consistent calculations according to a set of the Monte-Carlo kinetic models, we estimated the atmospheric loss rate of atomic oxygen caused by precipitation of $\mathrm{H}$-ENAs during proton aurorae on Mars. The obtained values, $(3.5-5.8) \times 10^{7} \mathrm{~cm}^{-2} \mathrm{~s}^{-1}$, are comparable to the atomic oxygen loss due to photochemical reactions (Groeller et al., 2014; Jakosky et al., 2018). It is worth noting that, though proton aurorae are sporadic events, the precipitation-induced flux of escaping hot oxygen atoms, according to the recent MAVEN observations (Deighan et al., 2015; Lee et al., 2015; Jakosky et al., 2018), may become dominant under conditions of extreme solar eventssolar flares and coronal mass ejections. It is currently supposed that the atmospheric neutral oxygen loss of Mars due to the impact of the solar wind plasma and, in particular, the precipitation of high-energy protons and hydrogen atoms during solar flares may play an important role in the atmospheric loss on astronomic time scales (Jakosky et al., 2018).

\section{FUNDING}

The study was supported by the Russian Scientific Foundation, project no. 19-12-00370 (Chapters FORMATION OF HOT OXYGEN ATOMS and MODEL RESULTS).

\section{OPEN ACCESS}

This article is licensed under a Creative Commons Attribution 4.0 International License, which permits use, sharing, adaptation, distribution and reproduction in any medium or format, as long as you give appropriate credit to the original author(s) and the source, provide a link to the Creative Commons licence, and indicate if changes were made. The images or other third party material in this article are included in the article's Creative Commons licence, unless indicated otherwise in a credit line to the material. If material is not included in the article's Creative Commons licence and your intended use is not permitted by statutory regulation or exceeds the permitted use, you will need to obtain permission directly from the copyright holder. To view a copy of this licence, visit http://creativecommons.org/licenses/by/4.0/.

\section{REFERENCES}

Chaffin, M.S., Chaufray, J.Y., Deighan, J., Schneider, N.M., McClintock, W.E., Stewart, A.I.F., et al., Elevated atmospheric escape of atomic hydrogen from Mars in- 
duced by high-altitude water, Nat. Geosci., 2017, vol. 10, pp. 174-178.

Chaffin, M.S., Chaufray, J.Y., Deighan, D., Schneider, N.M., Mayyasi, M., Clarke, J.T., et al., Mars H escape rates derived from MAVEN/IUVS Lyman alpha brightness measurements and their dependence on model assumptions, J. Geophys. Res.: Planets, 2018, vol. 123, pp. 2192-2210.

Chaufray, J.Y., Bertaux, J.L., Leblanc, F., and Quemerais, E., Observation of the hydrogen corona with SPICAM on Mars Express, Icarus, 2008, vol. 195, pp. 598-613.

Clarke, J.T., Bertaux, J.L., Chaufray, J.Y., Gladstone, G.R., Quemerais, E., Wilson, J.K., and Bhattacharyya, D., A rapid decrease of the hydrogen corona of Mars, Geophys. Res. Lett., 2014, vol. 41, pp. 8013-8020.

Deighan, J., Chaffin, M.S., Chaufray, J.-Y., Stewart, A.I.F., Schneider, N.M., Jain, S.K., Stiepen, A., Crismani, M., McClintock, W.E., Clarke, J.T., Holsclaw, G.M., Montmessin, F., Eparvier, F.G., Thiemann, E.M.B., Chamberlin, P.C., and Jakosky, B.M., MAVEN IUVS observation of the hot oxygen corona at Mars, Geophys. Rev. Lett., 2015, vol. 42, no. 21, pp. 9009-9014. https://doi.org/10.1002/2015GL065487

Deighan, J., Jain, S.K., Chaffin, M.S., Fang, X., Halekas, J.S., Clarke, J.T., Schneider, N.M., Stewart, A.I.F., Chaufray, J.-Y., Evans, J.S., Stevens, M.H., Mayyasi, M., Stiepen, A., Crismani, M., McClintock, W.E., Holsclaw, G.M., Lo, D.Y., Montmessin, F., Lefevre, F., and Jakosky, B.M., Discovery of a proton aurora at Mars, Nat. Astron., 2018, vol. 2, pp. 802-807.

Fedorova, A., Bertaux, J.L., Betsis, D., Montmessin, F., Korablev, O., Maltagliati, L., and Clarke, J., Water vapor in the middle atmosphere of mars during the 2007 global dust storm, Icarus, 2018, vol. 300, pp. 440-457.

Fedorova, A.A., Montmessin, F., Korablev, O., Luginin, M., Trokhimovskiy, A., Belyaev, D.A., and Wilson, C.F., Stormy water on Mars: The distribution and saturation of atmospheric water during the dusty season, Science, 2020, vol. 367, pp. 297-300.

Fox, J.L. and Hać, A.B., Photochemical escape of oxygen from Mars: A comparison of the exobase approximation to a Monte Carlo method, Icarus, 2009, vol. 204, no. 2 , pp. 527-544.

Halekas, J.S., Seasonal variability of the hydrogen exosphere of Mars, J. Geophys. Res.: Planets, 2017, vol. 122, pp. 901-911.

Halekas, J.S., McFadden, J.P., Connerney, J.E.P., Espley, J.R., Brain, D.A., Mitchell, D.L., Larson, D.E., Harada, Y., Hara, T., Ruhunusiri, S., and Jakosky, B.M., Timedispersed ion signatures observed in the Martian magnetosphere by MAVEN, Geophys. Rev. Lett., 2015, vol. 42, no. 21, pp. 8910-8916.

https://doi.org/10.1002/2015GL064781

Heavens, N.G., Kleinboehl, A., Chaffin, M.S., Halekas, J.S., Kass, D.M., Hayne, P.O., et al., Hydrogen escape from Mars enhanced by deep convection in dust storms, Nat. Astron., 2018, vol. 2, no. 2, pp. 126-132.

Hughes, A., Chaffin, M., Mierkiewicz, E., Deighan, J., Jain, S., Schneider, N., Mayyasi, M., and Jakosky, B.M., Proton aurora on Mars: A dayside phenomenon pervasive in southern summer, J. Geophys. Res.: Space Phys., 2019, vol. 124, pp. 10533-10548.

Groeller, H., Lichtenegger, H., Lammer, H., and Shematovich, V.I., Hot oxygen and carbon escape from the Martian atmosphere, Planet. Space Sci., 2014, vol. 98, pp. 93-105.
Jakosky, B.M., Grebowsky, J.M., Luhmann, J.G., Connerney, J., Eparvier, F., Ergun, R., Halekas, J., Larson, D., Mahaffy, P., McFadden, J., Mitchell, D.F., et al., MAVEN observations of the response of Mars to an interplanetary coronal mass ejection, Science, 2015, vol. 350 , no. 6261 , art. id. aad0210.

Jakosky, B.M., Brain, D., Chaffin, M., Curry, S., Deighan, J., and Zurek, R., Loss of the Martian atmosphere to space: Present-day loss rates determined from MAVEN observations and integrated loss through time, Icarus, 2018, vol. 315, pp. 146-160.

Lee, Y., Combi, M.R., Tenishev, V., Bougher, S.W., Deighan, J., Schneider, N.M., McClintock, W.E., and Jakosky, B.M., A comparison of 3-d model predictions of Mars' oxygen corona with early MAVEN IUVS observations, Geophys. Rev. Lett., 2015, vol. 42, no. 21, pp. $9015-9022$.

https://doi.org/10.1002/2015GL065291

Lillis, R.J., Brain, D.A., Bougher, S.W., Leblanc, F., Luhmann, J.G., Grebowsky, J., et al., Characterizing atmospheric escape from Mars today and through time with MAVEN, Space Sci. Rev., 2015, vol. 195, pp. 357442.

Lillis, R.J., Deighan, J., Fox, J.L., Bougher, S.W., Lee, Y., Combi, M.R., Cravens, T.E., Rahmati, A., Mahaffy, P.R., Benna, M., Elrod, M.K., McFadden, J.P., Ergun, R.E., Andersson, L., Fowler, C.M., Jakosky, B.M., Thiemann, E., Eparvier, F., Halekas, J.S., Leblanc, F., and Chaufray, J-Y., Photochemical escape of oxygen from Mars: First results from MAVEN in situ data, J. Geophys. Res.: Space Phys., 2017, vol. 122, pp. 3815-3836.

Maltagliati, L., Montmessin, F., Korablev, O., Fedorova, A., Forget, F., Maattanen, A., et al., Annual survey of water vapor vertical distribution and water-aerosol coupling in the Martian atmosphere observed by SPICAM/MEX solar occultations, Icarus, 2013, vol. 223, no. 2, pp. 942-962.

Ritter, B., Gerard, J.-C., Hubert, B., Rodriguez, L., and Montmessin, F., Observations of the proton aurora on mars with SPICAM on board Mars Express, Geophys. Res. Lett., 2018, vol. 45, pp. 231-236.

Shematovich, V.I., Suprathermal particles in astrochemistry, Russ. Chem. Rev., 2019, vol. 88, pp. 1013-1045.

Shematovich, V. I. and Kalinicheva, E.S., Atmospheric escape of oxygen atoms during the proton aurorae at Mars, Astron. Rep., 2020, vol. 64, pp. 628-635.

Shematovich, V.I., Bisikalo, D.V., Gerard, J.-C., and Hubert, B., Kinetic Monte Carlo model of the precipitation of high-energy proton and hydrogen atoms into the Martian atmosphere with taking into account the measured magnetic field, Astron. Rep., 2019, vol. 63, pp. 835-845.

Shematovich, V.I., Bisikalo, D.V., and Zhilkin, A.G., Effects of column density variations of extended hydrogen corona of mars on the charge exchange efficiency with solar wind protons, Astron. Rep., 2021, vol. 65, pp. 203208.

Vandaele, A.C., Korablev, O., Daerden, F., Aoki, S., Thomas, I.R., Altieri, F., and Zorzano, M.P., Martian dust storm impact on atmospheric $\mathrm{H}_{2} \mathrm{O}$ and $\mathrm{D} / \mathrm{H}$ observed by ExoMars Trace Gas Orbiter, Nature, 2019, vol. 568, pp. 521-525.

Translated by E. Petrova 